\title{
Video Article \\ Live-Cell Imaging Assays to Study Glioblastoma Brain Tumor Stem Cell Migration and Invasion
}

\author{
Ian Restall ${ }^{1}$, Danielle Anne Bozek ${ }^{1}$, Charles Chesnelong ${ }^{1,2}$, Samuel Weiss ${ }^{1}$, H. Artee Luchman ${ }^{1}$ \\ ${ }^{1}$ Hotchkiss Brain Institute, Department of Cell Biology and Anatomy, Cumming School of Medicine, University of Calgary \\ ${ }^{2}$ Department of Developmental and Stem Cell Biology Program, Arthur and Sonia Labatt Brain Tumor Research Centre, The Hospital for Sick Children
}

Correspondence to: H. Artee Luchman at aluchman@ucalgary.ca

URL: https://www.jove.com/video/58152

DOI: doi: $10.3791 / 58152$

Keywords: Cancer Research, Issue 138, Glioblastoma (GBM), brain tumor stem cell (BTSC), migration, invasion, glioma, cancer stem cell (CSC), live-cell imaging

Date Published: 8/29/2018

Citation: Restall, I., Bozek, D.A., Chesnelong, C., Weiss, S., Luchman, H.A. Live-Cell Imaging Assays to Study Glioblastoma Brain Tumor Stem Cell Migration and Invasion. J. Vis. Exp. (138), e58152, doi:10.3791/58152 (2018).

\section{Abstract}

Glioblastoma (GBM) is an aggressive brain tumor that is poorly controlled with the currently available treatment options. Key features of GBMs include rapid proliferation and pervasive invasion into the normal brain. Recurrence is thought to result from the presence of radio- and chemoresistant brain tumor stem cells (BTSCs) that invade away from the initial cancerous mass and, thus, evade surgical resection. Hence, therapies that target BTSCs and their invasive abilities may improve the otherwise poor prognosis of this disease. Our group and others have successfully established and characterized BTSC cultures from GBM patient samples. These BTSC cultures demonstrate fundamental cancer stem cell properties such as clonogenic self-renewal, multi-lineage differentiation, and tumor initiation in immune-deficient mice. In order to improve on the current therapeutic approaches for GBM, a better understanding of the mechanisms of BTSC migration and invasion is necessary. In GBM, the study of migration and invasion is restricted, in part, due to the limitations of existing techniques which do not fully account for the in vitro growth characteristics of BTSCs grown as neurospheres. Here, we describe rapid and quantitative live-cell imaging assays to study both the migration and invasion properties of BTSCs. The first method described is the BTSC migration assay which measures the migration toward a chemoattractant gradient. The second method described is the BTSC invasion assay which images and quantifies a cellular invasion from neurospheres into a matrix. The assays described here are used for the quantification of BTSC migration and invasion over time and under different treatment conditions.

\section{Video Link}

The video component of this article can be found at https://www.jove.com/video/58152/

\section{Introduction}

Glioblastoma (GBM) is the most common and aggressive form of brain cancer and, despite the current therapy involving surgical resection followed by radiation and chemotherapy, the median survival of patients is a mere 15 months ${ }^{1}$. GBM is invasive, highly drug resistant, and ultimately, an incurable disease that requires continued research into its inherent biology in order to identify novel therapeutic avenues. The high mortality rate of GBM patients is primarily caused by the extensive cellular invasion into adjacent normal brain tissue and the migration along blood vessels, which leads to a recurrence of the disease following treatment ${ }^{2,3}$. A subset of cells, termed brain tumor stem cells (BTSCs), that are slow cycling and therapeutically resistant, have been hypothesized to lead to disease recurrence. GBM BTSCs display properties of clonogenic self-renewal, multipotency, and tumor-initiating potential ${ }^{4,5,6}$. BTSCs also retain the genetic, molecular, and phenotypic heterogeneity of their parent GBM tumors ${ }^{7,8,9,10}$. These properties make BTSCs an extremely useful model system for the study of GBM and for exploring novel therapeutic strategies. In vivo, these stem-like cells are capable of tumor formation, growth, and invasion when implanted into the brains of neonatal rats ${ }^{11}$ and non-obese diabetic/severe combined immunodeficiency (NOD/SCID) mice ${ }^{4}$. The capability of BTSCs to repeatedly form invasive tumors in vivo that recapitulate the cellular and histological characteristics of their original tumors strongly supports their role as tumorinitiating cells ${ }^{12}$.

Novel therapeutic approaches are being developed to target BTSCs and help improve the long-term outcome for GBM patients. Currently, there is limited understanding of the cellular migration and invasion processes that are associated with therapeutic resistance and recurrence. Migration and invasion are distinct phenomena; migration is the process underlying the directed movement of cells and involves the reorganization of the cytoskeleton, adhesion molecules, and assembly/disassembly of focal contact points, whereas invasion also requires the physical destruction of barriers such as an extracellular matrix ${ }^{13}$. A widely accepted methodology of studying cell migration and invasion is the Boyden Chamber assay ${ }^{14,15}$. For this technique, a double chamber is filled with media, with the media in the bottom chamber supplemented with a chemoattractant. Chemotaxis is triggered using specific growth factors or cytokines, or fetal bovine serum as a non-specific chemoattractant. Following the gradient of the chemoattractant, cells migrate through the porous membrane. At the endpoint, the migrated cells can be visualized and quantified on the bottom of the membrane by staining with cytological or fluorescent dyes. The Boyden migration assay can be modified to an invasion assay by coating the porous filter with extracellular matrix components such as type I collagen or a basement membrane-like matrix. Invasive cells degrade the matrix, move through to the bottom of the porous filter, and can be quantified in a similar manner to the 
migration assay. Other commonly used migration assays include the scratch wound and cell exclusion zone assays ${ }^{13}$. The scratch wound assay is performed by scratching a gap in a cellular monolayer and observing the migration of cells from the edge of the wound by imaging at regular intervals until the scratch is closed. In the cell exclusion assay, a physical barrier is used to create a cell-free zone prior to the seeding of cells. The barrier is removed once the cells are confluent and the migration of the cells into the cell-free zone is imaged regularly ${ }^{16}$.

These previously established assays are frequently used for studying the migration or invasion of the adherent and homogeneous cell populations but pose considerable disadvantages when studying GBM BTSCs or other heterogeneous cancer cell populations. The Boyden Chamber assay can only generate endpoint measurements versus a kinetic assessment of cell movement. An observation over time is of high relevance for the measurement of BTSC migration, given that cells from different cultures often migrate at different rates. As such, the conditions and timing of the assay must be optimized for each culture type and requires time-intensive labor for the adequate sampling and quantification. The scratch and cell exclusion assays are not well-suited to BTSC cultures as, even when BTSCs are cultured under monolayer conditions on laminin-coated plates, we have observed that BTSCs appear to resist movement into the open space and prefer to stay in close proximity to other cells. Furthermore, these established migration assays do not allow for the visualization and monitoring of individual cells throughout an experiment. The monitoring of individual cells over time is valuable for the assessment of migration in heterogeneous cell populations such as BTSCs. Additional disadvantages of the Boyden chamber, scratch, and cell-exclusion zone assays for BTSC cultures are that they require relatively high cell numbers, can be time-consuming to set up, and either rapidly equilibrate or do not have a chemoattractant gradient. As such, these assays are not ideal to use for rare or slow-growing cell populations or for drug screening. Furthermore, these assays are not suited for measuring an invasion in a three-dimensional (3D) format, which is especially important for BTSCs grown under neurosphere conditions.

Here, we describe assays specifically modified for the observation and quantification of the migration for individual BTSCs, and for the invasion of GBM BTSCs cultured as neurospheres. The first assay describes an adaptation of the Boyden chamber assay using live-cell time-lapse imaging and a chemotaxis migration plate to measure chemotactic cell migration ${ }^{13}$. Live-cell imaging in a multi-well format allows for the visualization and quantification of cell migration under multiple treatment conditions. The second assay described here is a spheroid invasion assay ${ }^{13,17}$, which measures the invasive properties of BTSCs cultured under neurosphere conditions and embedded into a 3D extracellular matrix under various treatment conditions. Overall, these assays are much more compatible than previously described methodologies for studying the migratory and invasive properties of heterogeneous BTSC cultures. They also offer better opportunities for the investigation of novel therapeutic strategies to target both migration and invasion, which contribute significantly to disease recurrence and lethality.

\title{
1. Culturing Brain Tumor Stem Cells Previously Derived from Human Glioblastoma Specimens
}

\author{
Note: BTSC cultures were previously established from human GBM patient samples ${ }^{6,7,8,9,10}$.
}

1. Thaw a vial of cryogenically preserved BTSCs in a beaker containing $70 \%$ ethanol, placed inside a water bath at $37{ }^{\circ} \mathrm{C}$, just until the last of the ice has thawed. Dilute the thawed cells in $10 \mathrm{~mL}$ of media in a $15 \mathrm{~mL}$ conical tube and centrifuge the cells at 150 relative centrifugal force (RCF) for 7 min.

Note: Throughout these protocols, complete media refers to standard media used to culture BTSCs (previously described by Kelly et al.) ${ }^{6}$, typically with growth factors present. Media without growth factors refers to the base media with all components but without any growth factors supplemented.

2. Aspirate the media, resuspend the cells in $8 \mathrm{~mL}$ of complete media, and transfer the cells and media to a T25 flask.

3. After 1 week of culturing under non-adherent conditions, monitor the flask daily and supplement the contents with $1-2 \mathrm{~mL}$ of complete media as needed, if the media begins to deplete or starts to become acidic as indicated by an orange-yellow color change. Passage cells when the neurospheres reach a diameter of approximately $250 \mu \mathrm{m}$, typically between $10-14 \mathrm{~d}$ (Figure 1).

Note: The doubling time of different BTSC cultures varies widely. Neurosphere size can be measured using an ocular micrometer.

4. To passage BTSCs, collect the media and neurospheres by pipetting the media down the surface of the flask in order to collect all neurospheres and transfer them to a $15 \mathrm{~mL}$ conical tube and centrifuge the tube for $7 \mathrm{~min}$ at $150 \mathrm{RCF}$. Note: Flasks can be checked under a microscope to ensure that all neurospheres have been collected. An additional wash with $5 \mathrm{~mL}$ of phosphate buffered saline (PBS) or media can be performed to collect any remaining neurospheres as necessary.

5. To dissociate the BTSC neurospheres into single cells, aspirate the media and add $1 \mathrm{~mL}$ of pre-warmed cell detachment solution and incubate that at $37^{\circ} \mathrm{C}$ for $7 \mathrm{~min}$. Triturate the BTSC suspension $40 \mathrm{x}$ with a P1,000 micropipette set at $800 \mu \mathrm{L}$. Note: Cells can be incubated longer at $37^{\circ} \mathrm{C}$ if the neurospheres do not dissociate.

6. Add $5 \mathrm{~mL}$ of PBS (with the antibiotics penicillin and streptomycin) to the dissociated BTSCs and centrifuge the cells at $150 \mathrm{RCF}$ for 7 min.

7. Aspirate the supernatant and resuspend the BTSCs in 1-4 mL of complete media, depending on the size of the cell pellet. Note: The cell suspension needs to be further diluted if the concentration is beyond the accurate detection limit for accurate cell counting.

8. Using a dye that excludes dead cells, such as trypan blue, count the number of viable cells and seed the BTSCs into the flask or plate of interest-typically, 200,000 - 300,000 cells in $8 \mathrm{~mL}$ of complete media in a T25 flask.

\section{Brain Tumor Stem Cell Migration Assay}

1. Seed 200,000 cells in a T25 flask in $8 \mathrm{~mL}$ of complete media 1 - 2 weeks before planning to perform a migration assay. Note: The time between plating and performing the assay will vary depending on the growth rate of the BTSC culture used.

1. To test the effect of a drug treatment on migration, pretreat the cells with the appropriate vehicle, such as DMSO, and drug of interest by adding applicable volumes of the vehicle or drug stock directly into separate wells of media containing the cells.

2. Prepare the chemotaxis migration plate (Figure 2A) by coating it with a thin layer of collagen. 
1. Calculate the number of wells of a 96-well chemotaxis plate that will need to be coated with collagen. Include at least three replicate wells for each condition.

2. Dilute $5 \mathrm{mg} / \mathrm{mL}$ of stock of type I collagen to $0.2 \mathrm{mg} / \mathrm{mL}$ in pure acetic acid diluted to $0.14 \%$ in culture grade water.

3. Pipette $0.2 \mathrm{mg} / \mathrm{mL}$ of collagen to both the membrane insert wells and the bottom reservoir wells that are being used.

Note: The bottom reservoirs of the plate require $225 \mu \mathrm{L}$ and the membrane insert wells require $75 \mu \mathrm{L}$ of collagen for a proper coating.

4. Place the migration plate in an incubator at $37^{\circ} \mathrm{C}$ for $1 \mathrm{~h}$. Aspirate the collagen solution from the wells without scratching the collagen coating.

5. Wash the wells $2 x$ with sterile $1 x$ PBS. If the plate is not being used right away, aspirate the PBS and store it at $4{ }^{\circ} \mathrm{C}$ for up to 2 weeks. Prior to use, warm the chemotaxis migration plate to room temperature.

3. Prepare the bottom reservoir of the chemotaxis migration plate.

1. Pipette $225 \mu \mathrm{L}$ of media (without growth factors), supplemented with $10 \%$ FBS as a chemoattractant, to the bottom reservoir wells of the chemotaxis migration plate.

2. Gently place the membrane insert into the bottom reservoir at an angle to avoid creating bubbles.

4. Plate the cells into the membrane insert of the chemotaxis migration plate.

1. Resuspend dissociated BTSCs in media (without growth factors) at a cell density of $50,000 \mathrm{cells} / \mathrm{mL}$. Add drugs to the media if assessing migration during different drug treatments. Alternately, seed equal numbers of pre-treated cells and control cells, while maintaining the final drug concentration when plating.

2. Plate $50 \mu \mathrm{L}$ of the BTSCs from step 2.4.1 into each well of the migration plate under the desired treatment conditions.

5. Image the chemotaxis migration.

1. Place the chemotaxis migration plate into the live-cell imaging system and set the automated imaging software to acquire microscopic images of the plate every $1-2 \mathrm{~h}$ for up to $72 \mathrm{~h}$. Using commercial software (see Table of Materials), right-click on the timeline and set the scan interval to every $2 \mathrm{~h}$ for $24 \mathrm{~h}$.

Note: The time interval and total elapsed time will vary between BTSC cultures used; start with $2 \mathrm{~h}$ intervals for $72 \mathrm{~h}$ until experience has been gained with individual BTSC cultures. If an automated imaging system is unavailable, then images of the same field of view can be acquired manually with a microscope and a camera connected to the imaging software.

2. Once the image acquisition is complete, obtain the images for the entire experiment and create a processing definition using the analysis software that differentiates cells from the background of the membrane and the migration pores (Figure 2B, red mask). Using commercial software (see Table of Materials), select a New Processing Definition and set the processing definition settings for BTSCs to a seed threshold of 52, a grow threshold of 85 , adjustments of the size (pixels) by -1 , and a minimum area of $200 \mu m^{2}$. Apply this processing definition to the bottom side of the membrane. Modify this analysis if it does not accurately distinguish cells from the background membrane.

3. Collect data as the cell surface area on the bottom side of the membrane for each of the three replicate wells, which only counts the cells that have migrated through the pores. Graph the migration of the cells in $\mu^{2}$ (area of cells migrated) over time (Figure 2C,

Figure 3).

Note: Ensure that the relative number of cells plated and counted on the top surface of the membrane at the first time-point are similar (less than $20 \%$ variation across all wells) between all treatment conditions to avoid any effects of uneven plating. Data collection using the commercial software (see Table of Materials) can be executed by launching the processing definition created in step 2.5.2, clicking on Metrics, then on Graph/export, and then on Data export.

\section{Brain Tumor Stem Cell Neurosphere Invasion Assay}

1. Seed 200,000 cells in $8 \mathrm{~mL}$ of complete media in a T25 flask 1 - 2 weeks before planning to perform an invasion assay.

Note: The timing from plating to performing the assay will depend on the proliferation rate of the BTSC culture used.

1. For pretreated cells, add the compound of interest to the flask at the desired concentration for the predetermined time course before the neurospheres are ready to be plated for invasion.

2. Wait until the average neurosphere size is approximately $150-200 \mu \mathrm{m}$; typically, this takes $7-14 \mathrm{~d}$, depending on the BTSC culture being used.

Note: It is typical to observe a distribution of sphere sizes in the flask.

3. Tip and gently mix the flask with a pipette and move $500 \mu \mathrm{L}$ of the BTSC neurospheres to a $1.5 \mathrm{~mL}$ conical tube for each treatment condition; this will be used to plate three replicate wells.

Note: Steps 3.3.1 - 3.3.2 are optional but recommended for the first invasion experiment on a new BTSC culture.

1. To determine the density of the neurospheres that will end up in the well, prepare a $1.5 \mathrm{~mL}$ tube as in step 3.3. Gently mix the neurospheres and move $100 \mu \mathrm{L}$ into a separate 96-well plate and allow the neurospheres to settle by gravity for 5 min.

2. Check the number of neurospheres under the microscope to ensure multiple neurospheres are in the region of the well that will be imaged without overcrowding it.

Note: Invading neurospheres require enough room to triple in diameter without interacting with neighboring neurospheres. The number of neurospheres per well can be adjusted by counting the number of neurospheres per well and adding more or fewer neurospheres from the T25 flask to the $1.5 \mathrm{~mL}$ conical tube in step 3.3 .

4. Place the $1.5 \mathrm{~mL}$ conical tube with the cells from step 3.3 on ice and perform steps $3.5-3.7$ on ice.

5. Let the neurospheres settle by gravity to the bottom of the $1.5 \mathrm{~mL}$ conical tube for $5 \mathrm{~min}$ and prechill a labeled 96-well plate on ice.

6. Resuspend the neurospheres in the extracellular matrix protein.

1. Prepare $500 \mu \mathrm{L}$ of a $0.4 \mathrm{mg} / \mathrm{mL}$ type I collagen solution on ice for each treatment condition: add $459 \mu \mathrm{L}$ of media (without growth factors), $40 \mu \mathrm{L}$ of the $5 \mathrm{mg} / \mathrm{mL}$ collagen stock solution, and $0.92 \mu \mathrm{L}$ of sterile $1 \mathrm{~N} \mathrm{NaOH}$ (Table 1). 
Note: $500 \mu \mathrm{L}$ of the collagen solution is required per treatment condition: $100 \mu \mathrm{L}$ for each of the three replicate wells and $200 \mu \mathrm{L}$ of excess. Drug treatments need to be added here at the desired final concentration, subtracted from the media component of the solution. This protocol describes the use of type I collagen as the extracellular matrix protein; however, any extracellular matrix protein can be tested. In addition, for BTSC cultures that have slower rates of invasion, growth factors or FBS can be supplemented in the media to increase the rate of invasion.

2. After the neurospheres from step 3.5 have settled, aspirate as much of the media as possible without losing the neurosphere pellet that has settled at the bottom. Gently resuspend the neurospheres in $500 \mu \mathrm{L}$ of the collagen solution prepared in step 3.6.1.

7. Add $100 \mu \mathrm{L}$ of the collagen and neurosphere mixture to three replicate wells of the 96 -well plate on ice. Allow the neurospheres to settle on ice for $5 \mathrm{~min}$.

8. Transfer the 96 -well plate to a $37^{\circ} \mathrm{C}$ incubator for 5 min to allow for collagen polymerization

9. Immediately prepare to image the 96 -well plate.

1. Place the 96-well plate into the tray of a live-cell imaging system or on the stage of a microscope to acquire an image as a reference for the neurosphere size at the time of plating, before the invasion begins.

2. Acquire images every hour until the rate of invasion has plateaued; typically, this occurs within $24 \mathrm{~h}$.

Note: The imaging interval can be modified depending on the equipment being used. The imaging interval and total elapsed time can also be modified for BTSC cultures that have different rates of invasion.

10. Determine the increasing surface area of BTSCs as they invade the matrix over time.

Note: The surface area of cells at the time of plating, calculated as the mean of three replicate wells, needs to be similar (less than $20 \%$ variation across all wells) between the different treatment conditions to ensure the differences in the BTSC invasion are not strongly affected by the number or size of the neurospheres plated.

1. For the live-cell analysis system, use a 10X objective for the image acquisition and acquire four images per well for the three replicate wells. To determine the relative cell surface area, represented as the percent confluence of the well, set up a processing definition that specifically highlights the cells over the background of the well. Using commercial software (see Table of Materials), select a New Processing Definition and set the segmentation adjustment to 0.8 , the area minimum to $300 \mu \mathrm{m}^{2}$, and the eccentricity maximum to 0.99. Modify this analysis if it does not accurately distinguish cells from the background.

Note: If using other methods to image neurosphere invasion over time, it is recommended to obtain multiple fields of view for the three replicate wells over time. However, not imaging the exact same field of view at each interval will increase the standard error of the data collected. Computer software can be used to help measure the surface area of the invading cells in each image.

2. Collect data as the total cell surface area (either relative or absolute) for every well at each time-point and determine the mean of the three replicate wells. Graph the invasion of BTSCs by plotting the increasing cell area over time.

Note: Data collection using commercial software (see Table of Materials) can be executed by launching the processing definition created in step 3.10.1, clicking on Metrics, then on Graph/export, and then on Data export.

\section{Representative Results}

Here, we describe examples of data that can be obtained using live-cell imaging assays to study BTSC migration and invasion. BTSCs are plated as single cells and can be grown as free-floating neurospheres (Figure 1). We show that BTSCs can be dissociated and plated in the wells of the membrane insert in a chemotaxis migration plate that is coated with a thin layer of type I collagen (Figure 2A). Individual BTSCs are evenly dispersed on the top of the membrane insert at the beginning of the assay. Over a period of $24-72 \mathrm{~h}$, cells adhere to the membrane, move along the collagen-coated surface, and migrate through one of the 96 small pores to the bottom side of the membrane, toward a chemoattractant in the reservoir well. After highlighting the cells on the top of the membrane in yellow, the pores in blue, and the cells on the bottom of the membrane in red, cells can be seen entering in the pore on the top (yellow cell approaching a blue pore) and exiting the pore on the bottom (red cell exiting the blue pore) (Figure 3). Importantly, cells can be drug-treated and plated in the same chemotaxis migration plate as vehicle-treated control cells to study the effect of therapeutic interventions on BTSC migration (Figure 2B). The chemotaxis migration plate allows for the imaging of cells both on the top and on the bottom of the membrane insert. Therefore, quantification of cell migration over time is possible by counting the cells that have migrated to the bottom side of the membrane (Figure $2 \mathrm{C}$ ).

We have found that if BTSCs are not fully dissociated, then cells do not migrate through the pores and remain as small neurospheres (Figure 4A). It is also important to not plate more than $2,500 \mathrm{BTSC}$ /well as this results in cell clumping rather than in migration to the bottom side of the membrane (Figure 4B). Lastly, it is essential to avoid making bubbles in the membrane insert wells when plating cells and when placing the membrane insert onto the bottom reservoir, as this prevents accurate imaging and quantification (Figure 4C).

Next, we show that BTSC invasion, from neurospheres into the surrounding matrix, can be imaged and quantified over time in a 96-well plate format (Figure 5A). Invasion can be measured by quantifying the surface area of the cells as they invade the matrix over time. Using image analysis software, a processing definition was applied that overlays a mask onto the cell surface area, which allows for automatic quantification over time (Figure 5B). Time-lapse videos of BTSC invasions can also be created, to easily observe the overall process of BTSC invasion (Figure 6). In addition, fluorescent microscopy can be used to image BTSCs that express fluorescent proteins, such as green fluorescent protein (GFP) or red fluorescent protein (RFP). Here, we have created a time-lapse video of BTSCs expressing a cytosolic form of GFP to help track individual BTSCs as they invade a basement membrane-like matrix (Figure 7). This BTSC neurosphere invasion assay also allows for drug treatments to be added in replicate wells to directly assess their effects on BTSC invasion from neurospheres into a matrix (Figure 8A). The quantification of multiple neurospheres in replicate wells can be calculated and graphed over time to assess the effect of drugs at multiple concentrations (Figure 8B). 
Failure to adhere to the protocol described above can lead to an inability to obtain reliable and accurate data. Embedding BTSC neurospheres that are too large, with a diameter greater than $200 \mu \mathrm{m}$, leads to a plane of focus that is too large to accurately quantify all spheres in a field of view (Figure 8C). Similarly, failure to maintain the matrix at $4{ }^{\circ} \mathrm{C}$ while embedding the neurospheres can also lead to spheres that are not on the same plane of focus and an extracellular matrix that has not solidified evenly, which prevents the collection of accurate data (Figure 8D).
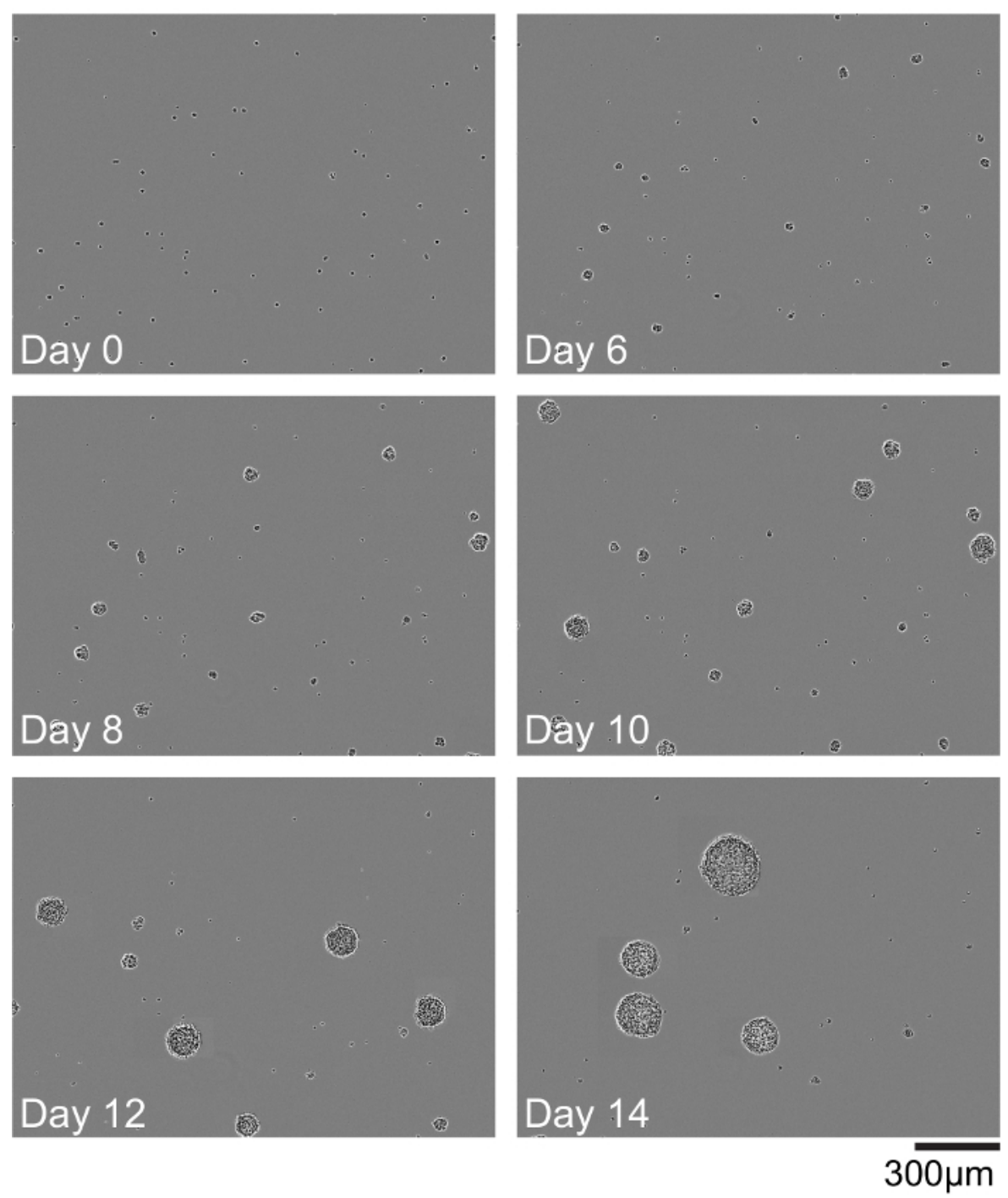

Figure 1: Culturing BTSCs. These panels are a representative example of BTSCs grown from single cells to neurospheres over a 14-d period in culture. The neurospheres shown here at day 14 are of an appropriate size for passaging. Please click here to view a larger version of this figure. 

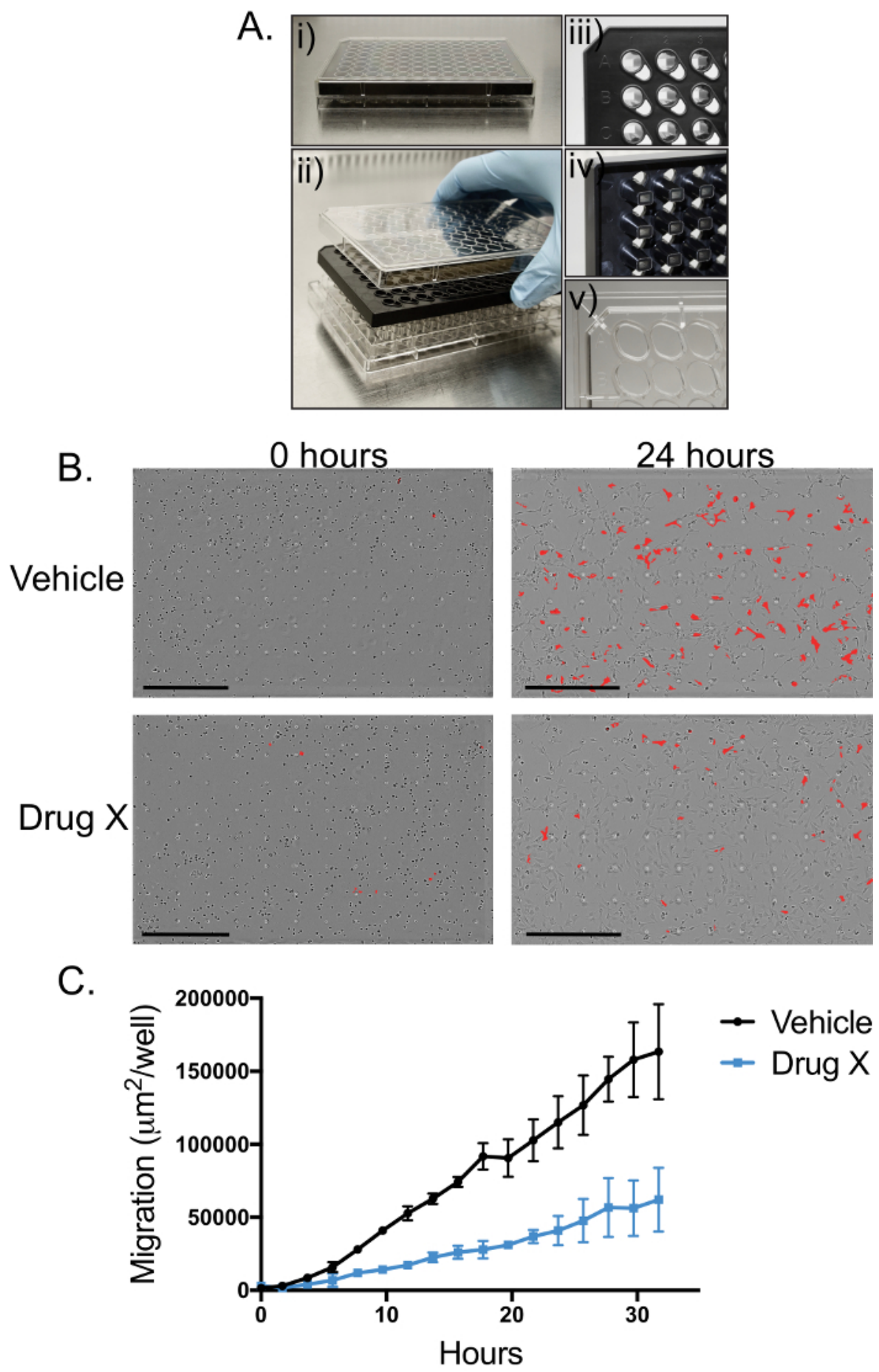

Figure 2: Live-cell imaging of a BTSC migration. (A) This is an example of a 96-well chemotaxis migration plate coated with a thin layer of type I collagen prior to performing the migration assay with BTSCs. The images show i) a plate with all three parts (lid, membrane insert, and reservoir) placed together, ii) all three parts separated, iii) a close-up image of the top view of the membrane insert, iv) a close-up image of the bottom view of the membrane insert, and v) a close-up image of the bottom reservoir. (B) These are representative images of a BTSC migration at the start $(0 \mathrm{~h})$ of the experiment and at $24 \mathrm{~h}$. The image focus is on the top of the membrane insert where the cells were originally plated. Cells that have migrated to the bottom of the chemotaxis migration plate are highlighted in red. At $0 \mathrm{~h}$, a few cells have migrated to the bottom side of the membrane. After $24 \mathrm{~h}$, the number of migrated cells has increased dramatically. The comparison of the images at $24 \mathrm{~h}$ for cells treated with a vehicle vs. drug X demonstrates that the drug treatment decreases BTSC migration. The scale bars represent $600 \mu \mathrm{m}$. (C) This panel shows the quantification of a BTSC migration following pre-treatment with a vehicle or drug X. The graph shows that the drug treatment has a strong effect on the migration of BTSCs. The data points are the mean of three technical replicate wells, and the error bars represent standard deviation (SD). Please click here to view a larger version of this figure. 


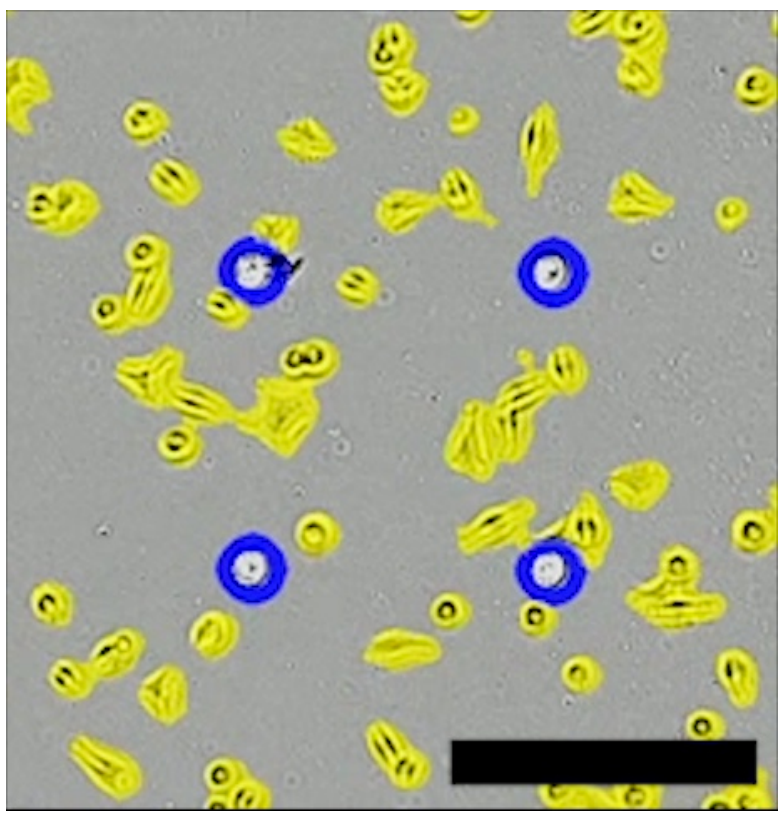

Animated Figure 3: BTSC migration time-lapse video. This video shows the migration of BTSCs using a type I collagen-coated chemotaxis migration plate. Cells on the top of the membrane insert are highlighted in yellow, cells that have migrated to the bottom of the membrane insert are highlighted in red, and the pores in the membrane are highlighted in blue. The plane of focus is set to image cells on the bottom side of the membrane. The video was created using time-lapse images taken every hour for $51 \mathrm{~h}$ and compiled at 4 frames per second. The scale bar represents $200 \mu \mathrm{m}$. Please click here to view this video. (Right-click to download.) 
A.

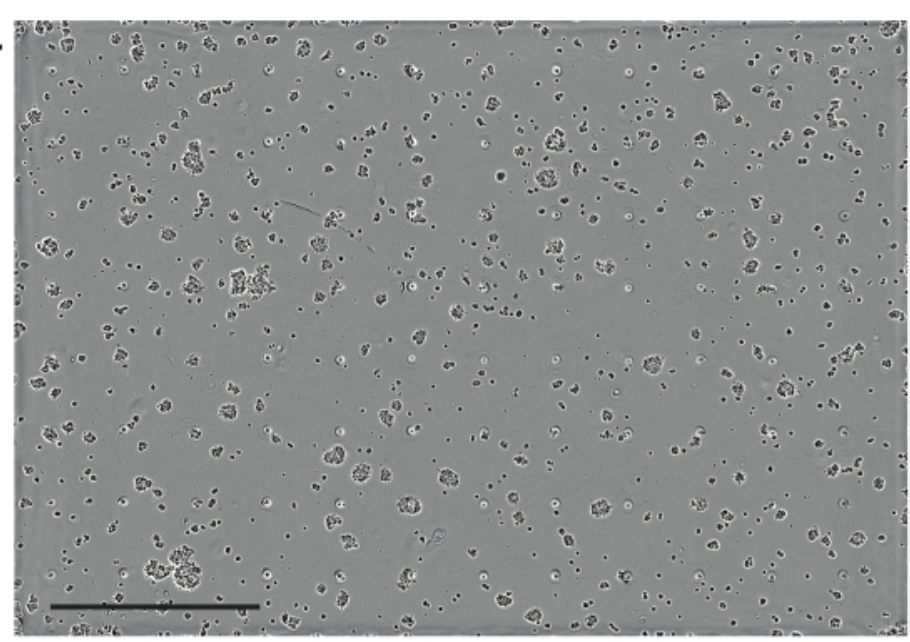

B.
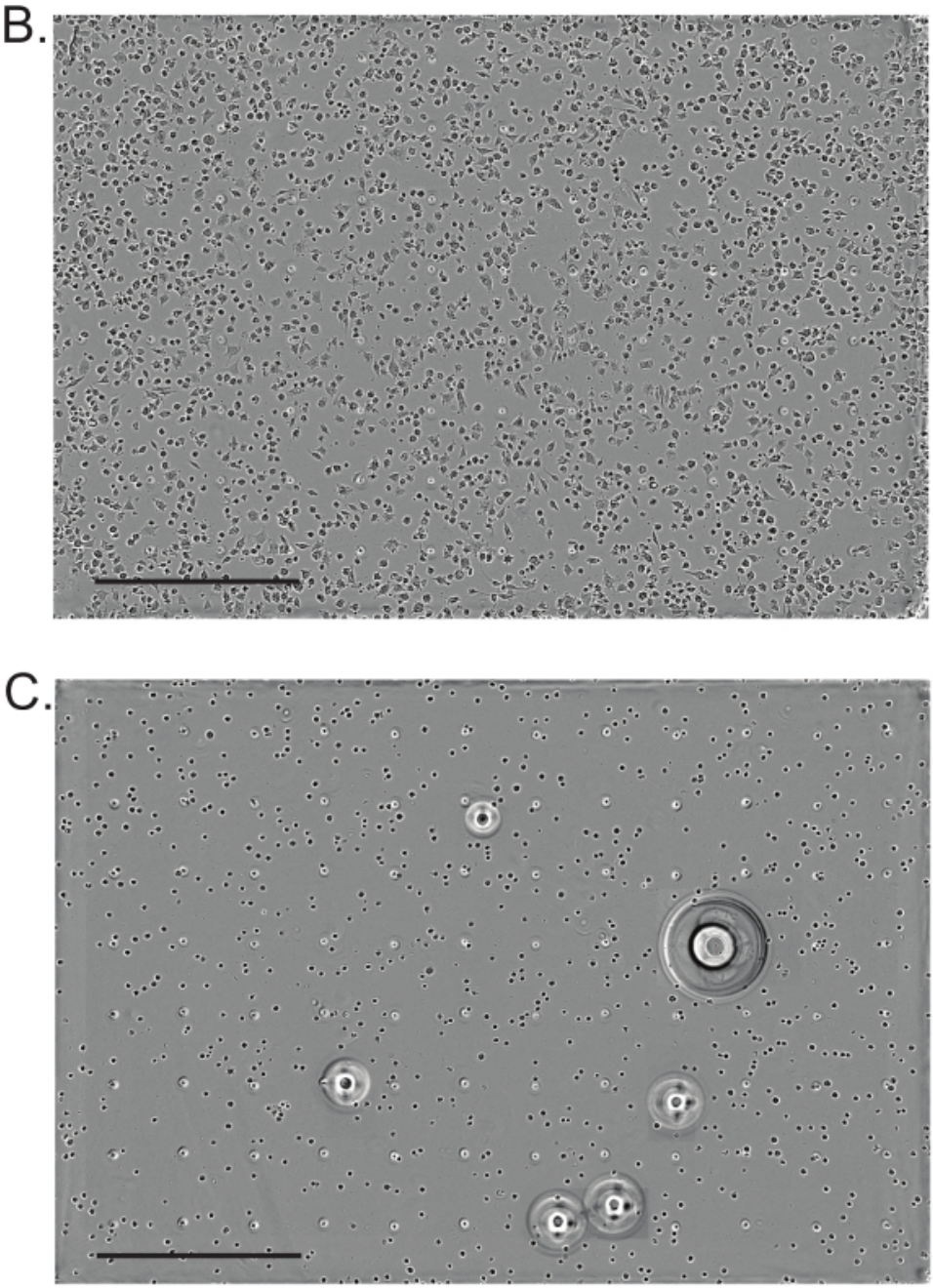

Figure 4: Examples of errors in the migration assay set-up. (A) This panel shows an example of BTSCs that were not fully dissociated prior to plating on the top membrane insert of the chemotaxis migration plate. Due to the large size of the spheres and the small size of the membrane pores, the cells are unable to migrate to the bottom reservoir. (B) This panel shows an example of a migration assay where too many cells were plated. The clumping of the cells interferes with cell migration and impairs quantification. (C) This panel shows an example of bubble formation on the membrane insert where BTSCs were plated. Bubbles result in poor image quality and prevent an accurate quantification of migration. The scale bars represent $600 \mu \mathrm{m}$. Please click here to view a larger version of this figure. 
A. Phase contrast
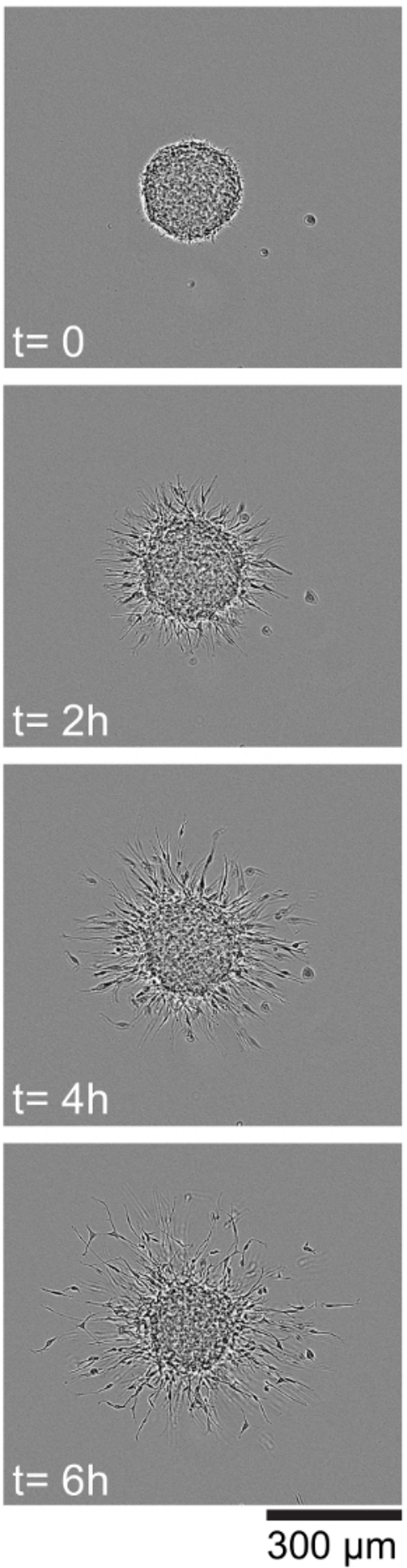

B.

Mask
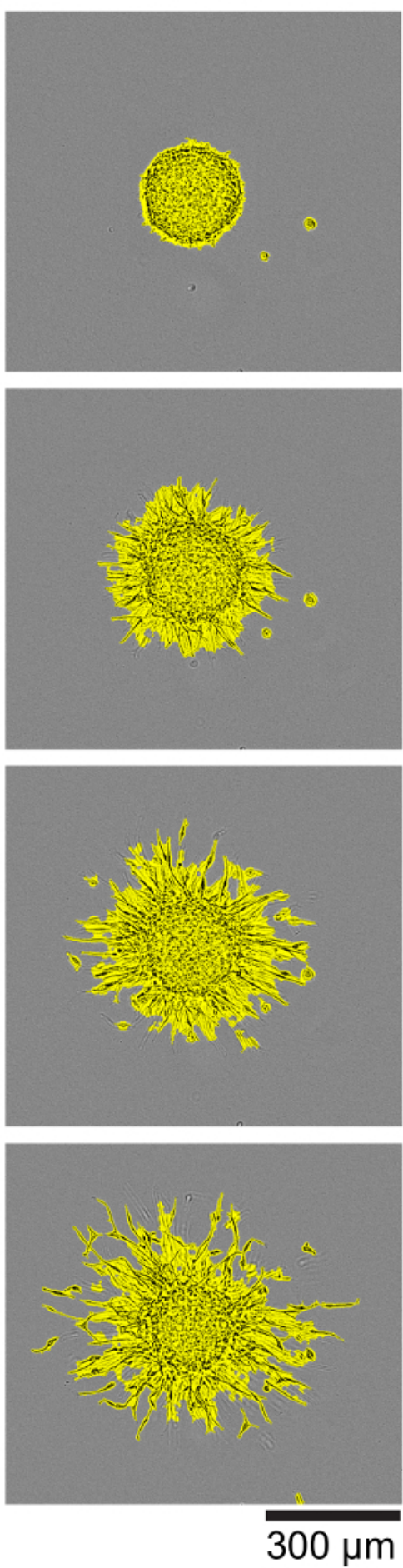

Figure 5: BTSC neurosphere invasion assay. These panels show an example of BTSCs invading from a neurosphere into a $0.4 \mathrm{mg} / \mathrm{mL}$ type I collagen matrix over a $6 \mathrm{~h}$ period. Here, (A) phase contrast images at each time-point are shown (B) with the representative quantitative mask overlaid, as described in detail in protocol step 3. Please click here to view a larger version of this figure. 


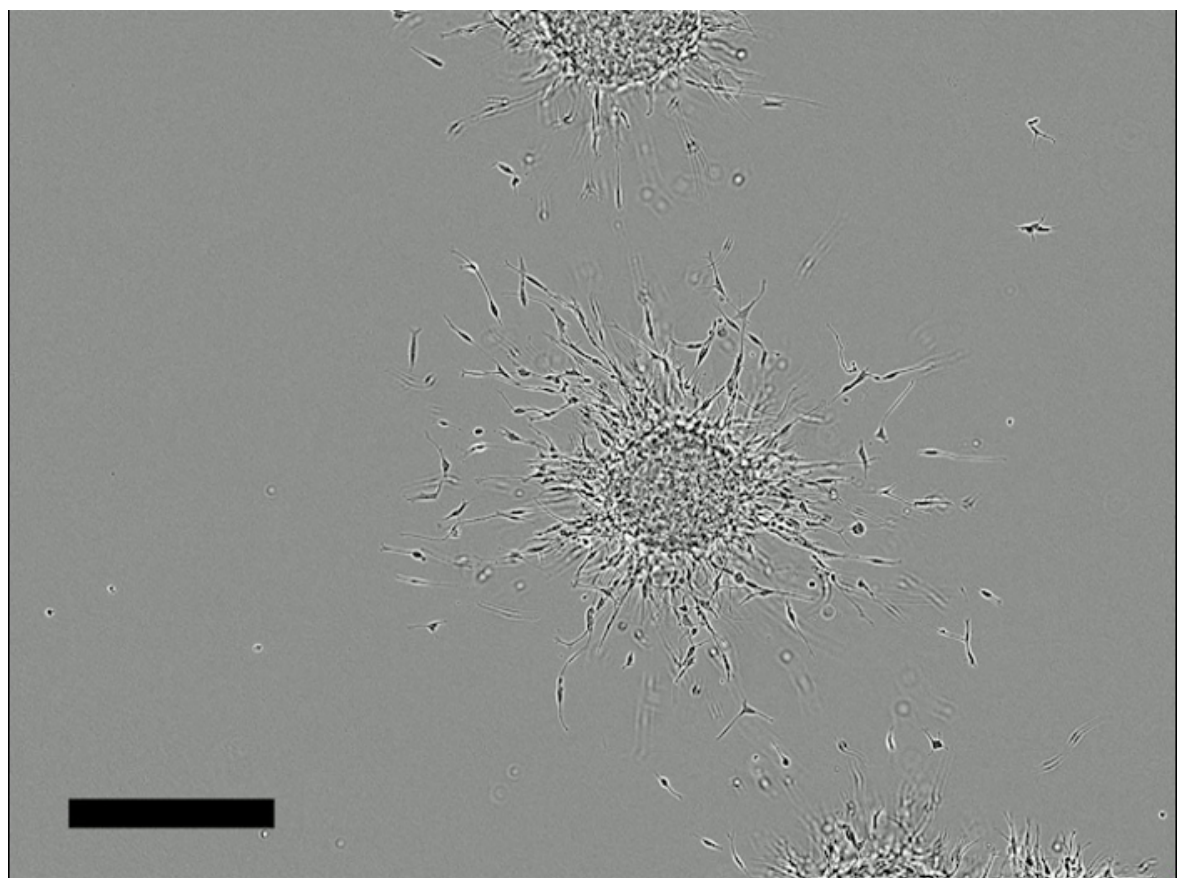

Figure 6: BTSC neurosphere invasion time-lapse video in type I collagen. This video shows an invasion of BTSCs from a neurosphere into a $0.4 \mathrm{mg} / \mathrm{mL}$ type I collagen matrix (also shown as still images in Figure 5A). The images were acquired using a 10X objective every hour for 12 $\mathrm{h}$ and are compiled at 4 frames per second. The scale bar represents $300 \mu \mathrm{m}$. Please click here to view this video. (Right-click to download.)

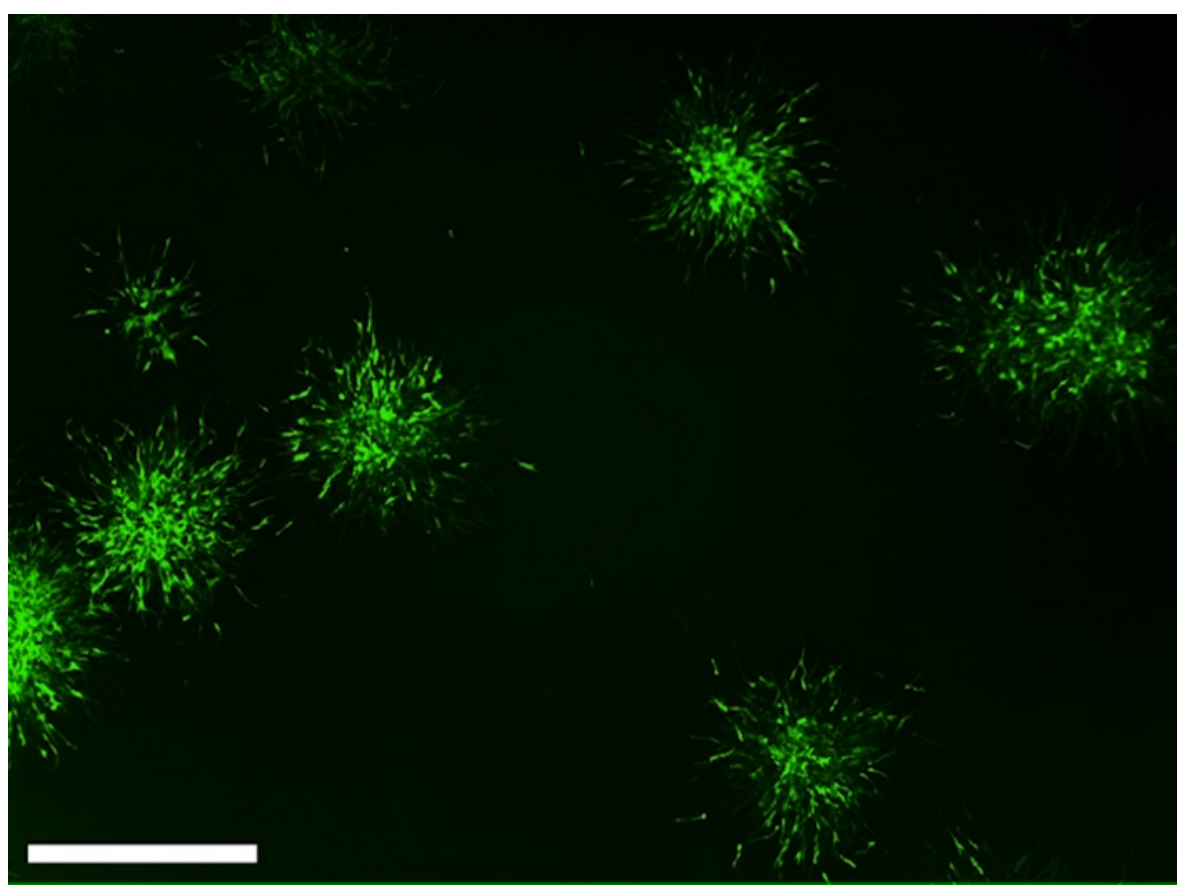

Figure 7: BTSC neurosphere invasion time-lapse video in a basement membrane-like matrix. This video shows an invasion of BTSCs, that express cytosolic GFP, from a neurosphere into a basement membrane-like matrix. The images were acquired using a $4 \mathrm{X}$ objective every 30 min for $48 \mathrm{~h}$ and were compiled at 8 frames per second. The scale bar represents $800 \mu \mathrm{m}$. Please click here to view this video. (Right-click to download.) 
A.
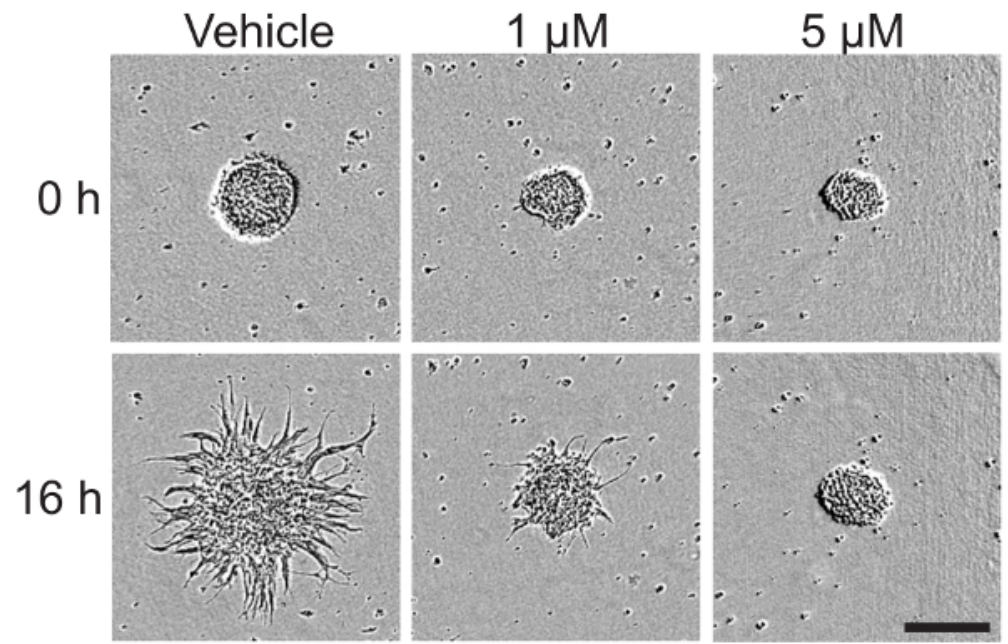

B.

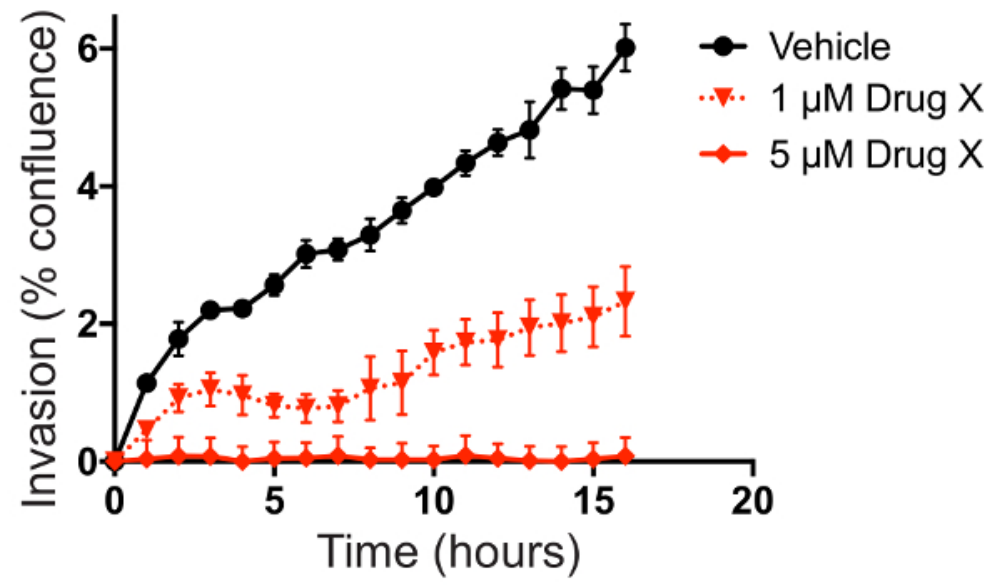

C.

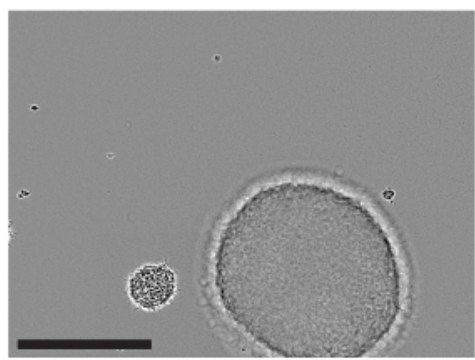

D.

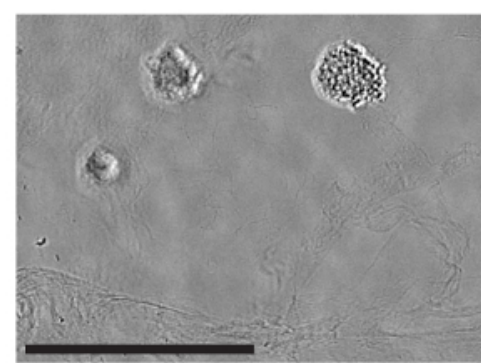

Figure 8: BTSC neurosphere invasion assay with treatment. (A) BTSC neurospheres were embedded in a basement membrane-like matrix prepared with vehicle or the indicated concentrations of drug X. Images were acquired every hour; shown here are images taken at the time of plating and after $16 \mathrm{~h}$. The scale bar represents $200 \mu \mathrm{m}$. (B) An invasion of BTSCs from neurospheres into the surrounding matrix was quantified every hour, as described in protocol step 3 . The data points are the mean of three technical replicate wells, and the error bars represent the standard error of the mean (SEM). (C) This panel shows BTSC neurospheres embedded in type I collagen with a sphere that is too large for quantification. The scale bar represents $300 \mu \mathrm{m}$. (D) This panel shows BTSC neurospheres embedded in type I collagen that did not fully set. The scale bar represents $300 \mu \mathrm{m}$. Please click here to view a larger version of this figure. 


\begin{tabular}{|l|l|}
\hline Collagen solution preparation & 5 \\
\hline Stock [Collagen] $(\mathrm{mg} / \mathrm{ml})$ & 0.4 \\
\hline Final [Collagen] $(\mathrm{mg} / \mathrm{ml})$ & 5 \\
\hline Number of wells needed: & 500 \\
\hline Total volume $(\mu \mathrm{l})$ & 40 \\
\hline Volume of stock collagen $(\mu \mathrm{l})$ & 0.92 \\
\hline Volume of $1 \mathrm{~N} \mathrm{NaOH}(\mu \mathrm{l})$ & 459 \\
\hline Volume of media $(\mu \mathrm{l})$ & \\
\hline
\end{tabular}

Table 1: Preparation of the $0.4 \mathrm{mg} / \mathrm{mL}$ type I collagen solution. Listed are the known starting and desired final concentrations of the type I collagen solution. The volumes listed are sufficient to plate neurospheres into three replicate wells, plus two for excess, and can include a single treatment condition, as described in detail in protocol step 3.

\section{Discussion}

Given that cancer cells are actively proliferating, this poses a potential technical challenge for the study of cell migration. For the migration assay described here, BTSCs are plated without growth factors, which limits the proliferation, and the chemoattractant gradient of the chemotaxis plate does not fully equilibrate over 72 hours. In addition, as the cells are being monitored using live-cell imaging over time, cells can be visually monitored to ensure widespread cell division is not occurring. For the success of the migration assay, it is important to dissociate the neurospheres completely to single cells and accurately count the cell numbers prior to plating. Plating spheres or plating too many cells, which results in clumping, can prevent cells from migrating through the small pores (Figure 4A and 4B). It is also important to avoid creating bubbles when plating the cells in the chemotaxis migration plate or when placing the membrane insert onto the bottom reservoir. Bubbles can alter the plane of focus and prevent accurate quantification (Figure 4C). The entire plate needs to be coated with a thin layer of collagen because this gives cells a surface to navigate on to the pores in the plate, as this assay requires cells to adhere to and then migrate across a biologically relevant surface towards the chemoattractant.

The BTSC migration protocol described here allows for the kinetic assessment of individual cell migration, requiring fewer cells for testing different treatment conditions when compared to existing protocols. This is important for cultures that grow very slowly, as it can be difficult to collect a large number of cells for experimentation. The low pore density of the chemotaxis migration plate allows for a slower equilibration of the chemoattractant gradient, greater than $72 \mathrm{~h}$, and more effectively mimics the biological process of chemotactic migration over a longer period of time. The protocol was optimized to have a very thin layer of collagen coating on the chemotaxis migration plate to ensure that the assay measures migration and not invasion. It also facilitates imaging and the quantification of single cells as they adhere to the collagen matrix coating the membrane insert. However, one limitation of the technique is that the quantification of the migration across multiple wells over time is greatly simplified and expedited using commercial software (see Table of Materials). A notable advantage of the migration assay as described here is the wide adaptability of the protocol to other cell types. It will be especially useful for quantifying the chemotactic migration of cell lines that are either slow growing, difficult to adhere, or migrate slowly.

To ensure the success of the BTSC invasion assay, it is necessary to collect neurospheres before they become larger than $200 \mu \mathrm{m}$. Larger spheres have a plane of focus that is too large to consistently image and quantify (Figure 5C). Furthermore, neurospheres can begin to clump and aggregate with other neurospheres. This affects the acquisition of accurate and consistent data. In addition, it is essential that the neurospheres are allowed to settle on the bottom of the well while the collagen is still on ice, to allow the neurospheres to be imaged in a single plane of focus (Figure 5D). Similarly, it is important to allow the collagen to fully set without moving the plate as this can disrupt the formation of an even matrix, which might negatively affect the image quality (Figure 5D). For the BTSC invasion assay described here, one of the major advantages is that BTSCs cultured as neurospheres can be assessed for invasion directly, without the need to be grown adherently. Furthermore, BTSC neurospheres can be embedded in any extracellular matrix, either using individual components of the extracellular matrix from a specific tissue or using a commercially available basement membrane mixture. Specifying the extracellular matrix can help to identify or test specific mechanisms of invasion. For example, with this protocol, it is possible to assess the effect of targeting individual members of the matrix metallopeptidase family of genes, which are known to degrade specific matrix components. Alternatively, although this protocol is specific to BTSC neurospheres, any cells grown as spheres can be used in a similar fashion. Examples include breast cancer cells cultured as mammospheres or primary colorectal cancer cells cultured as tumorspheres; however, this limits the technique to cell types that can grow as spheres in a culture. Other advantages of both the migration and invasion assays are that they are easy to set up, they work in a 96-well format, and the data is quantifiable over time.

Overall, in order to prevent post-treatment GBM recurrence, it is essential to develop methodologies that facilitate the investigation of BTSC migration and invasion. The migration protocol described here offers many advantages over previously established migration assays, especially for the study of GBM BTSCs. This improved migration protocol involves dissociating BTSCs cultured under neurosphere conditions and plating single cells in a 96-well collagen-coated chemotaxis migration plate. Using a live-cell imaging system, the migration of BTSCs can be monitored and quantified over time. The invasion assay described here allows for the monitoring of BTSC invasions from neurospheres into a matrix. This assay involves embedding neurospheres into a collagen matrix, or another protein-rich extracellular matrix, in a 96-well plate. Imaging of the plate with a live-cell time-lapse imaging system allows for the analysis of BTSC invasions under different treatment conditions over time. These assays, hence, provide a valuable tool to scientists hoping to better understand the mechanisms underlying BTSC migration and invasion. 


\section{Disclosures}

The authors have nothing to disclose.

\section{Acknowledgements}

The authors thank Rozina Hassam and Orsolya Cseh for their technical support. This research project was supported in part by grants from the Canada Foundation for Innovation (to H. Artee Luchman and Samuel Weiss) and the Stem Cell Networks of Canada (also to H. Artee Luchman and Samuel Weiss).

\section{References}

1. Stupp, R., et al. Radiotherapy plus concomitant and adjuvant temozolomide for glioblastoma. New England Journal of Medicine. 352 (10), 987-996 (2005).

2. Holland, E.C. Glioblastoma multiforme: the terminator. Proceedings of the National Academy of Sciences. 97 (12), $6242-6244$ (2000).

3. Heddleston, J.M., et al. Glioma stem cell maintenance: the role of the microenvironment. Current Pharmaceutical Design. 17 (23), $2386-2401$ (2011).

4. Singh, S.K., et al. Identification of human brain tumour initiating cells. Nature. 432 (7015), 396-401 (2004).

5. Galli, R. Isolation and Characterization of Tumorigenic, Stem-like Neural Precursors from Human Glioblastoma. Cancer Research. 64 (19), 1-12 (2004).

6. Kelly, J.J.P., et al. Proliferation of Human Glioblastoma Stem Cells Occurs Independently of Exogenous Mitogens. STEM CELLS. 27 (8), 1722-1733 (2009).

7. Cusulin, C., et al. Precursor States of Brain Tumor Initiating Cell Lines Are Predictive of Survival in Xenografts and Associated with Glioblastoma Subtypes. Stem Cell Reports. 5 (1), 1-9 (2015).

8. Luchman, H.A., et al. Dual mTORC1/2 Blockade Inhibits Glioblastoma Brain Tumor Initiating Cells In Vitro and In Vivo and Synergizes with Temozolomide to Increase Orthotopic Xenograft Survival. Clinical Cancer Research. 20 (22), 5756-5767 (2014).

9. Jensen, K.V., Cseh, O., Aman, A., Weiss, S., Luchman, H.A. The JAK2/STAT3 inhibitor pacritinib effectively inhibits patient-derived GBM brain tumor initiating cells in vitro and when used in combination with temozolomide increases survival in an orthotopic xenograft model. PLoS ONE. 12 (12), e0189670 (2017).

10. Nguyen, S.A., et al. Novel MSH6 Mutations in Treatment-Naive Glioblastoma and Anaplastic Oligodendroglioma Contribute to Temozolomide Resistance Independently of MGMT Promoter Methylation. Clinical Cancer Research. 20 (18), 4894-4903 (2014).

11. Hemmati, H.D., et al. Cancerous stem cells can arise from pediatric brain tumors. Proceedings of the National Academy of Sciences. 100 (25), 15178-15183 (2003).

12. Lee, J., et al. Tumor stem cells derived from glioblastomas cultured in bFGF and EGF more closely mirror the phenotype and genotype of primary tumors than do serum-cultured cell lines. Cancer Cell. 9 (5), 391-403 (2006).

13. Menyhárt, O., et al. Guidelines for the selection of functional assays to evaluate the hallmarks of cancer. BBA - Reviews on Cancer. 1866 (2), 300-319 (2016).

14. Boyden, S. The chemotactic effect of mixtures of antibody and antigen on polymorphonuclear leucocytes. The Journal of Experimental Medicine. 115, 453-466 (1962).

15. Albini, A., et al. A Rapid in Vitro Assay for Quantitating the Invasive Potential of Tumor Cells. Cancer Research. 47 (12), $3239-3245$ (1987).

16. Liang, C.-C., Park, A.Y., Guan, J.-L. In vitro scratch assay: a convenient and inexpensive method for analysis of cell migration in vitro. Nature Protocols. 2 (2), 329-333 (2007).

17. Naber, H.P.H., Wiercinska, E., Dijke, ten, P., van Laar, T. Spheroid Assay to Measure TGF-ß-induced Invasion. Journal of Visualized Experiments. (57), e3337 (2011). 